\title{
Acidente Vascular Cerebral: o conhecimento dos enfermeiros
}

Recebido em: 12/12/2011

Aceito em: 16/04/2012

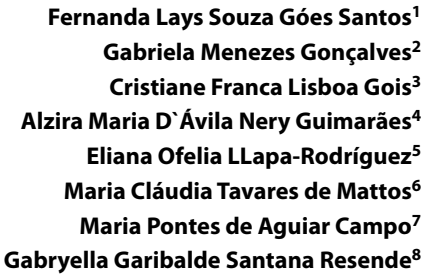

Entre as doenças que acometem o Sistema Nervoso Central, o Acidente Vascular Cerebral (AVC) é uma das mais prevalentes e de maior morbimortalidade. O estudo verificou o conhecimento dos enfermeiros sobre os fatores de risco, prevenção e prognóstico do AVC. Trata-se de um estudo descritivo, desenvolvido com 83 enfermeiros. Entre os participantes, $25,3 \%$ reconheceram todos os fatores de risco, $82,9 \%$ identificaram as ações preventivas e $49,3 \%$ reconheceram os possíveis prognósticos do AVC. Conclui-se que a maioria dos enfermeiros identificou as ações preventivas, apesar de poucos terem identificado todos os fatores de risco.

Descritores: Acidente Vascular Cerebral, Enfermagem, Atenção Primária à Saúde.

\section{Stroke: the knowledge of nurses}

Among the diseases affecting the central nervous system, the stroke is one of the most prevalent and highest morbimortality. The study assessed the nurse's knowledge about risk factors, prevention and prognosis of stroke. This is a descriptive study, developed with 83 nurses. Among participants, $25,3 \%$ recognized all risk factors, $82,9 \%$ identified preventive actions and $49,3 \%$ recognized the potential predictors of stroke. We conclude that the majority of the nurses identified preventive actions, although just a few have identified all the risk factors.

Descriptors: Stroke, Nursing, Primary Health Care.

\section{Accidente cerebrovascular: el conocimiento de los enfermeros}

Entre las enfermedades que afectan al Sistema Nervioso Central, el Accidente Cerebrovascular (ACV) es uno de los más frecuentes y de mayor morbilidad y mortalidad. El estudio evaluó los conocimientos de los enfermeros de los factores de riesgo, la prevención y el pronóstico del ACV. Este es un estudio descriptivo, realizado con 83 enfermeros. Entre los participantes, 25,3\% reconocieron todos los factores de riesgo, el 82,9\% identificaron las medidas de prevención y el 49,3\% reconocieron los posibles pronósticos de lo ACV. Se concluye que la mayoría de las enfermeras identifico las acciones de prevención, aunque pocos han identificado todos los factores de riesgo.

Descriptores: Accidente Cerebrovascular, Enfermería, Atención Primaria de Salud.

\section{INTRODUÇÃO}

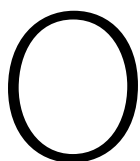
Acidente Vascular Cerebral (AVC) destaca-se entre as doenças que acometem o Sistema Nervoso Central (SNC) pela elevada prevalência e morbimortalidade, independentemente do nível de desenvolvimento do país(1). No Brasil, o AVC, não especificado se isquêmico ou hemorrágico, representa a terceira causa de maior internação e a segunda de maior número de mortes no conjunto das doenças que acometem o aparelho circulatório(2). Dos 86.994 óbitos que ocorreram no país em 2010 por doenças incluídas nesse grupo,
19.892 foram decorrentes de $\operatorname{AVC}^{(2)}$, o que o caracteriza como um problema de saúde pública a ser enfrentado.

Muitos são os fatores de risco descritos para o AVC, entre os quais se citam idade, hereditariedade, raça, hipertensão, diabetes melittus, dislipidemias, tabagismo e uso em excesso de álcool e drogas ${ }^{(3)}$, $\operatorname{cardiopatias}^{(4)}$, anticoncepcionais orais ${ }^{(5)} \mathrm{e}$ sedentarismo ${ }^{(6)}$.

A identificação e o controle dos fatores de risco são fundamentais na redução tanto do número de casos desse

1 Enfermeira especialista em Saúde da Família pela UGF-SP. Aracaju, Sergipe, Brasil. E-mail: fernandalays87@yahoo.com.br.

2 Enfermeira especialista em Saúde da Família pela UGF-SP, em Urgência e Emergência pela Unit e em Gestão das Organizações Públicas de Saúde pela UFS. Aracaju, Sergipe, Brasil.

3 Enfermeira, doutora, professor da Universidade Federal de Sergipe, São Cristovão, Sergipe, Brasil.

4 Enfermeira, doutora, professor da Universidade Federal de Sergipe, São Cristovão, Sergipe, Brasil.

5 Enfermeira, doutora, professora da Universidade Federal de Sergipe, São Cristovão, Sergipe, Brasil.

6 Enfermeira, doutora, professora da Universidade Federal de Sergipe, São Cristovão, Sergipe, Brasil.

7 Enfermeira, doutora, professora da Universidade Federal de Sergipe, São Cristovão, Sergipe, Brasil.

8 Enfermeira, especialista em Educação Profissional na Área de Saúde: enfermagem pela ENSP/Fiocruz; em Ativação de Processos de Mudança na Formação Superior de

Profissionais de Saúde pela ENSP/Fiocruz. Presidente do Coren-SE. Aracaju, Sergipe, Brasil. 
evento quanto na ocorrência de um novo evento ${ }^{(4)}$. As principais ações profissionais devem ser realizadas visando ao controle da pressão arterial, da diabetes, da dislipidemia, da fibrilação arterial, do controle do peso, abstinência de fumo e incentivo à prática de atividade física ${ }^{(7)}$.

Quanto ao prognóstico, a literatura menciona que o AVC pode causar várias alterações no indivíduo, como, por exemplo, distúrbio do desequilíbrio ${ }^{(8)}$, déficit motor ${ }^{(9)}$ e distúrbio de linguagem ${ }^{(10)}$.

A partir dos fatores de risco, são necessárias intervenções sustentadas com o objetivo de reverter os números alarmantes de AVC. Nesse sentido, os profissionais que atuam no Programa Saúde da Família (PSF) possuem papel singular, pois assistem à população na prevenção e no controle de doenças ${ }^{(11)}$. $O$ enfermeiro, como profissional que faz parte da Equipe de Saúde da Família (ESF), atua desenvolvendo, entre outras atividades, ações de educação em saúde. Sendo assim, é importante que possua conhecimento científico para atuar de forma apropriada no combate ao AVC.

De acordo com o apresentado e considerando o foco da Atenção Básica (AB), que é a promoção e a proteção da saúde, a prevenção de agravos, o diagnóstico, o tratamento, a reabilitação e a manutenção da saúde ${ }^{(12)}$, fica evidente a função dos profissionais da $A B$ no reconhecimento dos fatores de risco e no planejamento de ações que visem à redução do número de casos.

Diante do exposto e da escassez de artigos sobre AVC realizados no contexto do profissional enfermeiro, este estudo teve como objetivos verificar se os enfermeiros do PSF possuem conhecimento científico sobre os fatores de risco, prevenção e prognóstico para o AVC

\section{METODOLOGIA}

Trata-se de um estudo de caráter descritivo, do tipo corte transversal, desenvolvido em Unidades de Saúde da Família (USF) do município de Aracaju, estado de Sergipe. O município possui oito regiões, e cada uma conta com as USF correspondentes e suas respectivas ESF, perfazendo um total de 129 equipes. A equipe é formada, no mínimo, por médico, enfermeiro, auxiliar de enfermagem e agentes comunitários de saúde ${ }^{(12)}$.

A amostra foi composta de 97 enfermeiros que atenderam ao critério de inclusão: concordar em fazer parte da pesquisa. Tratase de uma amostra probabilística obtida através de um sorteio para saber quais as ESF, entre todas, fariam parte da amostra. A coleta dos dados foi realizada nos meses de maio a junho de 2010, após a aprovação do projeto pelo Comitê de Ética em Pesquisa da Universidade Tiradentes (protocolo no 250410). Os enfermeiros foram inicialmente informados quanto ao objetivo do estudo e, após concordância em participar, assinaram o Termo de Consentimento Livre e Esclarecido.

Para a coleta dos dados, foi utilizado um questionário composto de 13 questões objetivas, divididas em duas partes:

Parte I - dados sociodemográficos (sexo, idade, anos de graduação, pós-graduação, tempo de trabalho no PSF);

Parte II - dados referentes ao conhecimento dos enfermeiros sobre o AVC, experiência de atendimento à vítima de AVC, tipos de acidente, prevenção e possíveis prognósticos para o AVC.
Inicialmente, foi realizado um teste piloto com dez enfermeiros para verificar a adequação do instrumento aos objetivos do estudo. Após essa etapa, foram realizadas alterações no instrumento: introduziram-se na parte II, no que concerne às possíveis respostas às perguntas, algumas opções falsas (erradas), a fim de obter uma resposta que refletisse realmente o conhecimento investigado.

Os dados foram processados e analisados usando o programa Excel 2007. Para a análise descritiva, foram realizadas medidas de posição (média) e de dispersão (desvio-padrão) para as variáveis contínuas e de frequência simples para as variáveis categóricas.

\section{RESULTADO E DISCUSSÃO}

\section{Caracterização sociodemográfica}

Inicialmente, a amostra foi composta de 97 enfermeiros. Desses, seis não aceitaram participar do estudo, três estavam de férias, quatro de licença e um encontrava-se afastado sob atestado médico. Assim, a amostra final foi constituída de 83 enfermeiros.

Com relação à caracterização sociodemográfica dos participantes, a maioria era do sexo feminino e a média de idade foi de 42 anos. Quanto ao tempo de formação e atuação na área, a maior parte possuía mais de cinco anos de graduação e 57,8\% trabalhavam há mais de cinco anos no PSF. Dos enfermeiros participantes, $78,3 \%$ tinham curso de pós-graduação, especialização, residência e /ou mestrado (tabela 1).

Tabela 1 - Caracterização sociodemográfica dos 83 enfermeiros. Aracaju, 2010.

\begin{tabular}{|c|c|c|c|}
\hline Variável & No & $\%$ & M (D.P.) \\
\hline \multicolumn{4}{|l|}{ Sexo } \\
\hline Feminino & 73 & 87,9 & \\
\hline Idade (anos) & & & $42(9.27)$ \\
\hline \multicolumn{4}{|l|}{ Anos de Graduação } \\
\hline Mais de 5 anos & 75 & 90,3 & \\
\hline \multicolumn{4}{|l|}{ Tempo no PSF } \\
\hline Mais de 5 anos & 48 & 57,8 & \\
\hline Menos de 5 anos & 35 & 42,2 & \\
\hline \multicolumn{4}{|l|}{ Especialização } \\
\hline Sim & 65 & 78,3 & \\
\hline \multicolumn{4}{|l|}{ Área da Especialização* } \\
\hline $\begin{array}{l}\text { Sáude Coletiva/ Saúde Pública/ } \\
\text { Saúde da Família }\end{array}$ & 38 & 50,6 & \\
\hline Administração & 13 & 17,3 & \\
\hline Educação Pedagógica & 12 & 16,0 & \\
\hline Enfermagem do Trabalho & 06 & 8,0 & \\
\hline Obstetrícia & 04 & 5,3 & \\
\hline $\begin{array}{l}\text { Vigilância Epidemiológica/ } \\
\text { Sanitária }\end{array}$ & 04 & 5,3 & \\
\hline Urgência e Emergência & 03 & 4,0 & \\
\hline Residência em Cardiologia & 01 & 1,3 & \\
\hline Residência em Saúde da Família & 01 & 1,3 & \\
\hline Outros & 11 & 14,6 & \\
\hline
\end{tabular}


Os resultados deste estudo revelaram que todos os enfermeiros reconheceram o AVC como um distúrbio cerebrovascular, sendo que a maioria identificou os tipos de acidente. Com relação aos fatores de risco, 25,3\% dos enfermeiros identificaram todos os listados (tabela 2). Esse resultado chama a atenção, uma vez que os profissionais do PSF atuam no controle e na prevenção de doenças ${ }^{(11)}$, tendo, portanto, de deter conhecimento quanto aos fatores de risco, a fim de planejar ações que visem à redução do número de AVC e da mortalidade decorrente.

Quando questionados sobre as medidas de prevenção, 82,9\% dos enfermeiros assinalaram a opção que agrupava maior número de medidas. Foram incluídas duas respostas falsas (uso de analgésicos e de anticonvulsivante) e dois participantes marcaram essas opções como verdadeiras (tabela 2).

A melhor maneira de prevenir o AVC é identificando pacientes com maior propensão de desenvolver e controlar seus fatores de risco. Alguns fatores, como o fumo, podem ser eliminados; outros, como a hipertensão e estenose da aorta, podem ser controlados ou tratados para reduzir o risco ${ }^{(13)}$. Em pesquisa desenvolvida nos Estados Unidos, destaca-se que, embora os profissionais de saúde tenham conhecimento sobre os fatores de risco, a prevenção e o controle desses fatores continuam deixando a desejar ${ }^{(14)}$.

Inquérito domiciliar sobre comportamentos de risco e morbidade referida de doenças e agravos não transmissíveis realizado em 15 capitais e Distrito Federal entre 2002 e 2003 avaliou alguns fatores de risco para doenças crônicas não transmissíveis. Esse inquérito possibilitou identificar os fatores de risco frente à escolaridade, a qual constitui uma variável indiretamente relacionada à renda. Verificou-se que em todas as capitais o consumo do tabaco, a hipertensão e a diabetes estavam relacionados à menor grau de escolaridade e, com relação ao excesso de peso, na maioria das capitais também foi observada relação mais baixa de escolaridade ${ }^{(15)}$. Esses dados são importantes para os profissionais que atuam especialmente na $A B$, por assistirem a usuários que, em sua maioria, possuem menor poder aquisitivo e escolaridade.

Foram listados alguns prognósticos para vítimas de AVC (déficit de linguagem, déficit motor e perda do equilíbrio) e uma discreta minoria identificou todas as opções. São muitas as possíveis sequelas decorrentes do AVC, as quais acarretam dependência, sobretudo na fase inicial, sendo o déficit motor a mais facilmente identificada ${ }^{(16)}$.

Tabela 2 - Distribuição da frequência quanto ao conhecimento dos enfermeiros sobre AVC ( $n=83$ ). Aracaju, 2010

\section{Variável}

Conhecimento sobre o que é AVC

Distúrbio Cerebrovascular

Atendimento anterior à vítima de AVC

Sim

\section{Tipos de AVC}

Isquêmico

Isquêmico e hemorrágico

Outra opção (errada)

Fatores de Risco para o AVC

1 a 2

3 a 5

6 a 8

9 a 11

\section{Prevenção do AVC*}

Controle da PA

Controle da PA e alimentação saudável

Controle da PA e atividade física

Controle da PA, alimentação saudável e atividade física

Controle da PA, alimentação saudável, atividade física e uso de analgésicos e/ou uso de anticonvulsivantes

\section{Prognóstico do AVC**}

Déficit motor

Déficit motor e perda do equilíbrio

Déficit motor e de linguagem

Déficit motor, de linguagem e perda do equilíbrio

Outra opção (errada)

\begin{tabular}{|c|c|}
\hline No & $\%$ \\
\hline 83 & 100,0 \\
\hline 58 & 69,8 \\
\hline 09 & 10,8 \\
\hline 72 & 86,7 \\
\hline 02 & 2,4 \\
\hline 05 & 6,1 \\
\hline 12 & 14,4 \\
\hline 45 & 54,2 \\
\hline 21 & 25,3 \\
\hline 03 & 3,6 \\
\hline 05 & 6,0 \\
\hline 04 & 4,8 \\
\hline 68 & 82,9 \\
\hline 02 & 2,4 \\
\hline 06 & 7,4 \\
\hline 03 & 3,7 \\
\hline 30 & 37,0 \\
\hline 40 & 49,3 \\
\hline 02 & 2,4 \\
\hline
\end{tabular}

PA- Pressão Arterial $\quad{ }^{*} n=82 \quad{ }^{* *} n=81$ 
O AVC é uma doença que apresenta elevada incidência e possui grande potencial de causar incapacidades, as quais podem comprometer a qualidade de vida do indivíduo, da família e, numa análise mais ampla, do país. Isso porque o aumento do número depessoasacometidaseimpossibilitadas de exercer suas atividades laborais faz com que se tornem antecipadamente beneficiárias da Previdência Social. Para intervir nessa realidade, é necessário o desenvolvimento de ações proativas que de fato reduzam o número de casos de AVC e possibilitem melhor qualidade de vida para a população.

\section{CONCLUSÃO}

Conclui-se que a maioria dos enfermeiros identificou as ações preventivas, apesar de poucos terem identificado todos os fatores de risco.

Amparados na fundamentação teórica e na análise dos resultados do presente estudo, ressaltamos a necessidade de sensibilização dos enfermeiros para a importância do preparo no atendimento a usuários com risco de desenvolver um AVC, bem como a atualização continuada por meio da evidência científica produzida. Esse encaminhamento se faz premente, objetivando a redução do risco e da morbimortalidade por AVC. O estímulo e apoio à mudança são essenciais para que transformações aconteçam de fato. Por fim, é necessário que estudos avaliativos sejam conduzidos, para verificar a efetividade das intervenções adotadas e contribuir para a construção do conhecimento científico sobre o tema.

Algumas limitações devem ser mencionadas. Trata-se de estudo de corte transversal, o que limita considerações sobre causalidade. Outra limitação configura-se pela não-inclusão de mais variáveis que poderiam contribuir para uma avaliação mais ampla sobre o conhecimento científico dos enfermeiros. Contudo, as variáveis estudadas possibilitaram avaliar aspectos importantes mais relacionados à Atenção Básica.

\section{Referências}

1. Otero RM, Guimarães AG, Coelho PMP, Freitas GS. Terapia celular no acidente

vascular cerebral. Rev Bras Hematol Hemoter. 2009;31(5):99-103.

2. Ministério da Saúde (BR). Datasus: indicadores de saúde, 2010 [Internet]. [citado

em 2011 Jul 30]. Disponível em: http://tabnet.datasus.gov.br/cgi/tabcgi.exe?sih/

cnv/niuf.def

3. Sell FC. Accidente vascular cerebral? és el accidente vascular cerebral una

enfermedad tratable? Rev Costarric Cardiol. 2000;2(1):27-33.

4. Pires SL, Gagliardi RJ, Gorzoni ML. Estudo das frequências dos principais fatores de risco para acidente vascular cerebral isquêmico em idosos. Arq Neuropsiquiatr. 2004;62(3-B):844-51.

5. Zétola VHF, Nóvak EM, Camargo CHF, Júnior HC, Coral P, Muzzio JA, et al. Acidente vascular cerebral em pacientes jovens. Arq Neuropsiquiatr. 2001;59(3-B):740-5. 6. Biblioteca Virtual em Saúde [Internet]. [citado em 2012 Abr 08]. Disponível em: http://bvsms.saude.gov.br/bvs/dicas/105avc.html

7. Tambara EM. Diretrizes para atendimento pré-hospitalar no acidente vascular encefálico. In: Cavalcante IL, Cantinho FAF, Assad AR, organizadores. Medicina perioperatória. Rio de Janeiro: SAERJ; 2006. p. 77-83.

8. Michael KM, Shaughnessy M. Stroke prevention and management in older adults. J Cardiovasc Nurs. 2006;21(5 Suppl 1):S21-6.

9. Falcão IV, Carvalho EMF, Barreto KML, Lessa FJD, Leite VMM. Acidente vascular cerebral precoce: implicações para adultos em idade produtiva atendidos pelo Sistema Único de Saúde. Rev Bras Saúde Matern Infant. 2004;4(1):95-102.
10. Talarico TR, Venegas MJ, Ortiz KZ. Perfil populacional de pacientes com distúrbios da comunicação humana decorrentes de lesão cerebral, assistidos em hospital terciário. Rev CEFAC. 2011;13(2):330-9.

11. Pereira ABCNG, Alvarenga $H$, Pereira Jr RSP, Barbosa MTS. Prevalência de acidente vascular cerebral em idosos no Município de Vassouras, Rio de Janeiro, Brasil, através do rastreamento de dados do programa saúde da família. Cad Saúde Pública. 2009;25(9):1929-36.

12. Ministério da Saúde (BR). Portaria n²488/GM, de 21 de outubro de 2011 [Internet]. [citado em 2012 Abr 08]. Disponível em: https://docs.google.com/ file/d/0B5GcS2-UlzxjOGM10WY3NWMtYzcwOCOOYzQwLTkwOWMtOTQ5YWViMT IIOTY4/edit?pli=1

13. Sauerbeck LR. Primary stroke prevention. Am J Nurs. 2006;106(11):40-50.

14. Kozub E. Community stroke prevention programs: an overview. J Neurosci Nurs. 2010;42(3):143-9.

15. Ministério da Saúde (BR). Secretaria de Vigilância em Saúde. Secretaria de Atenção à Saúde. Instituto Nacional de Câncer. Coordenação de Prevenção e Vigilância. Inquérito domiciliar sobre comportamentos de risco e morbidade referida de doenças e agravos não transmissíveis: Brasil, 15 capitais e Distrito Federal, 2002-2003. Rio de Janeiro: INCA; 2004.

16. Brito ES, Rabinovich EP. A família também adoece: mudanças secundárias à ocorrência de um acidente vascular encefálico na família. Interface Comun Saúde Educ. 2008;12(27):783-94. 